\title{
Study on Construction of Expressway Traffic Safety Evaluation Model
}

\author{
Jun Chen ${ }^{1}$, Yamei Chen ${ }^{2}$ \\ ${ }^{1}$ Guiyang Water Resources and Transportation Development Investment Group Co., Ltd., Guiyang, \\ 550003, China \\ ${ }^{2}$ School of Civil Engineering, Guizhou University, Guiyang, 550003, China
}

Keywords: Expressway, Traffic safety, Evaluation model.

\begin{abstract}
With the constant increase in traffic amount and traffic mileage of expressways, the occurrence rate of expressway traffic safety accidents is also increasing, which brings about huge personal injury to the population. The government and relevant sectors pay high attention to this problem and indicate to solve it as soon as possible so as to guarantee people's travel safety. Hence, it is very necessary to construct expressway traffic safety evaluation model under current situation. The theme of this paper is to construct expressway traffic safety evaluation model. Firstly, the construction principle of expressway traffic safety evaluation system is discussed in this paper. Secondly, two basic evaluation models for expressway traffic safety are analyzed. Finally, corresponding countermeasures are given to ensure driving safety on expressways.
\end{abstract}

\section{Introduction}

With the rapid development of China's economy and the improvement of people's living standards, expressway mileage is on the rise in China. Meanwhile, people further pursue convenience and comfort. Almost every family owns the private car. Although national economy rises continuously, such a problem is confronted: the number of vehicles increases sharply, and traffic jam and even traffic accidents often happen. Besides, environmental pollution is caused by vehicles. All these become major factors influencing sustainable development of China. In order to safeguard life safety in a more comprehensive and timely way, it is required to find out the subjective and objective factors influencing expressway traffic safety, find out the law and construct corresponding expressway traffic safety evaluation index system. Finally, a complete and scientific expressway traffic safety evaluation model should be constructed.

\section{Construction of expressway traffic safety evaluation index system}

The reasons for expressway traffic accidents are various, so it is unreliable and unscientific to evaluate expressway traffic safety from one aspect. First of all, we must realize evaluation indexes of expressway traffic safety are various and diversified, and analyze and grasp them from each factor. Then, we should decide the evaluation criterion and implementation ways so as to achieve all-round evaluation of expressway traffic safety.

\section{To establish the principles of expressway traffic safety evaluation index system}

All-round, rational and scientific expressway traffic safety evaluation indexes have great significance and influence for scientific analysis of expressway traffic system. The selection of expressway traffic safety evaluation indexes generally should conform to the following principles:

Scientificity and reliability

The most important and also fundamental principle for evaluation indexes is scientificity, which is a fundamental requirement. If there is no factual basis for expressway traffic safety evaluation, actual situation cannot be reflected objectively, and there is relatively perfect and accurate evaluation theory, 
the evaluation makes no sense and does not have any guidance effect for practice. The evaluation index system should at least conform to fundamentals, general goal exception and requirement of expressway traffic safety engineering construction.

\section{Completeness}

Considering the factors influencing expressway traffic safety involve various aspects, the evaluation of expressway traffic safety should meet the requirement of diversification. Because it is one-sided and incomplete to reflect the features and functions of evaluation system from a perspective, it is required to combine practical level of expressway safety performance and embody dynamic trend and development change of expressway traffic safety system during constructing expressway traffic safety evaluation system.

Simpleness, explicitness and practicability

First of all, the purpose of constructing expressway traffic safety index system should be made clear, i.e. to guarantee expressway traffic safety, to discover potential safety hazards in time and to propose solutions. Thus, the principles that expressway traffic safety index system must follow are simpleness and practicability from the perspective of purposes. Therefore, during selection of index parameters, it is required to choose typical and representative data to ensure index fairness and acquire data in time.

\section{Independence}

Independence is mainly reflected in weighted sum. This is because during comprehensive evaluation of expressway traffic safety system, it is necessary to carry out weighted sum of weights of various indexes. These weights should keep independent, or else the distortion of comprehensive assessment result may be caused easily.

\section{To confirm expressway traffic safety evaluation index system}

To overall evaluate and analyze complex factors and indexes in expressway traffic safety system, it is necessary to confirm a complete expressway traffic safety evaluation index system. The evaluation system is composed of two parts: target layer and criterion layer. The target layer is microcosmic evaluation of expressway traffic safety. The criterion layer includes driver's behavior, driving environment, road conditions, vehicle performance and management level etc.

Driver's behavior

The influence of driver's behavior on expressway traffic safety is mainly reflected in overspeed driving, overload driving, drink-driving and other acts in violation of regulations. In combination of actual conditions, three factors may be chosen as the evaluation indexes of driver's behavior and quantified. The quantified indexes are overspeed rate, overload and ultralimit rate and average continuous driving time for the sake of follow-up statistics and calculation.

Vehicle performance

Usually, the main factors influencing vehicle performance include vehicle model and vehicle performance. According to the survey, vehicles on Chinese expressways are basically made in China. According to the principle of independence, performance evaluation can be made for vehicles on the expressway.

\section{Road conditions}

The analysis of factors influencing expressway traffic safety from the perspective of road conditions may be carried out from two aspects: plane and vertical section. Of course, anti-slide performance of road surface has the largest influence, and it is most closely related to weather change. In accordance with the independence principle, comprehensive and careful analysis and evaluation of expressway traffic safety caused by road conditions are implemented.

\section{Driving environment}

Driving environment contains many contents. For example, the weather, speed difference between cars and trucks and saturability etc. will result in severe influence on expressway traffic safety. The most representative evaluation indexes should be chosen on the basis of the practicability principle. 


\section{Expressway traffic safety evaluation model based on AHP-entropy}

The expressway traffic safety evaluation model based on AHP-entropy has obvious advantages so that index weight coefficient can take into account of subjective habit factor while meeting objectivity. How to establish the expressway traffic safety evaluation model based on AHP-entropy?

\section{Standardization of expressway traffic safety evaluation index data}

The expressway traffic safety evaluation index system based on AHP-entropy involves 12 second-level indexes in total. Besides, their original data have great numerical value differences and dimension differences. Thus, standardization is needed for the data, such as "Min-max" standardization method.

\section{Definition of weight coefficients for expressway traffic safety evaluation indexes}

How to confirm the weight coefficient of each index? AHP evaluation methodology may be adopted to confirm and analyze subjective weight of indexes. Meanwhile, the objective weight conformed through entropy weight method is combined to jointly confirm total weight coefficient of expressway traffic safety evaluation indexes based on AHP-entropy.

\section{Expressway traffic safety evaluation model based on AHP-entropy}

After the above steps are finished, the expressway traffic safety evaluation model based on AHP-entropy can be constructed. The basic construction process is as follows:

First of all, after subjective weight and objective weight are gained through AHP method and entropy weight method respectively, comprehensive weight coefficient is then confirmed. $\alpha_{\mathrm{f}}$ and $\omega_{\mathrm{f}}$ represent subjective weight and objective weight respectively. Then, $\alpha_{\mathrm{f}} \omega_{\mathrm{f}} / \sum_{\mathrm{f}=1}^{12}\left(\alpha_{\mathrm{f}} \omega_{\mathrm{f}}\right)$ is applied to gain comprehensive weight coefficient $\beta_{\mathrm{f}}$.

Secondly, the basic formula for construction of expressway traffic safety evaluation model based on AHP-entropy is as follows: $\vartheta \mathrm{e}=\sum_{\mathrm{f}=1}^{12} \beta \mathrm{fY}$ ef. In the formula, Y ef refers to the index value of percentage format of the fth evaluation index and the eth year, while $\vartheta$ e represents the evaluation value of the eth year.

\section{Expressway traffic safety evaluation model based on vector similarity}

Except the above expressway traffic safety evaluation model based on AHP-entropy, there is also a typical model system, i.e. the evaluation model based on vector similarity. Such model is different from the evaluation model based on AHP-entropy. It depends on the data after standardization, and utilizes vector relationship to expound the advantages and disadvantages of evaluation object. Finally, it reviews according to the similarity level between the evaluation object and the ideal value so as to construct the expressway traffic safety evaluation model based on vector similarity.

\section{Introduction to vector similarity}

Vector similarity refers to similarity level of two vectors after the comparison. Its numerical value is related to the distance between vectors and included angle cosine. Next, the author uses $\varsigma$ to represent vector similarity and applies $d$ to represent the distance between vectors. Meanwhile, $\cos \theta$ is used to represent included angle cosine between two vectors. So, the following formula can be obtained: $\varsigma=|\cos \theta| / 1+\mathrm{d}$. This formula may be used to calculate vector similarity to construct the model.

\section{Expressway traffic safety evaluation model based on vector similarity}

As discussed above, vector similarity is similarity level of two vectors, so vector similarity of expressway traffic safety represents the similarity between the ideal state of expressway traffic safety 
and safety condition. If expressway traffic safety level has an ideal value, the similarity between evaluation value and ideal value directly reflects the performance index and influence degree of the evaluation object. This degree can represent the expressway traffic safety degree. The specific operation steps are as follows:

Firstly, standardized evaluation matrix of expressway traffic safety evaluation indexes is confirmed. We set the evaluation object as $i=1,2,3, \ldots n$ and set the index as $j=1,2,3$, ...m. After standardization of the index $\mathrm{j}$, standardized evaluation matrix of expressway traffic safety can be gained, i.e. $\mathrm{P}_{\mathrm{ij}}=\left[\begin{array}{l}P 11 \ldots P 1 \mathrm{~m} \\ . P \mathrm{n} 1 \ldots . P \mathrm{~nm}\end{array}\right]$. In the formula, Pnm represents the value of the mth evaluation index in the nth year; Pij represents standardized evaluation matrix of expressway traffic safety.

Secondly, after the evaluation indexes are standardized, the weight coefficient of evaluation indexes should be confirmed next. AHP method may be adopted to confirm the weight coefficient of expressway traffic safety evaluation indexes and to analyze the influence of each evaluation index in the evaluation system. Here, the evaluation index is first expresses as j. Its weight coefficient is expressed as $\alpha \mathrm{j}$.

Thirdly, vector similarity of expressway traffic safety is calculated. The numerical value of expressway traffic safety in the nth year is deemed as a vector, and weighting evaluation is carried out for the vector, expresses as $\mathrm{Ai}$.

$$
\mathrm{Ai}=(\alpha 1 \mathrm{Pi} 1, \quad \alpha 2 \mathrm{Pi} 2, \ldots, \quad \alpha \mathrm{mPim})_{\mathrm{T}}
$$

A0 is set as the ideal value of evaluation. The weight coefficient of expressway traffic safety evaluation index is

$$
\mathrm{A} 0=(\alpha 1, \alpha 2, \ldots \alpha \mathrm{m})_{\mathrm{T}}
$$

Finally, based on the above formula, the formulas of the distance between vectors of expressway traffic safety and included angle cosine of vectors can be figured out: di=|Ai-A0|,

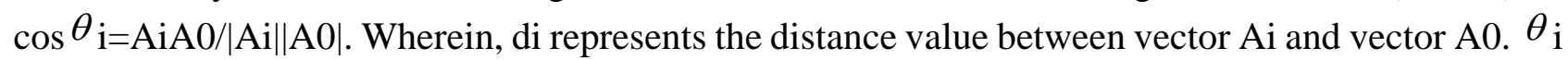
represents the included angle between $\mathrm{Ai}$ and $\mathrm{A} 0$.

By referring to the above formulas, vector similarity $\varsigma_{\mathrm{i}}$ of expressway traffic safety can be calculated:

$$
\varsigma_{\mathrm{i}=\mid \cos } \theta_{\mathrm{i} \mid / 1+\mathrm{di}}
$$

Based on the above analysis and discussion, the safety level of expressway at different time can be judged according to vector similarity $\varsigma_{\mathrm{i}}$ of expressway traffic safety. In the end, the reference data are organized and sorted.

\section{Study on relevant countermeasures}

Through constructing and analyzing expressway traffic safety evaluation system and evaluation model, we can roughly know comprehensive governance and solution should be done form the following aspects in order to practically solve expressway traffic safety issue.

Firstly, improve expressway traffic safety management system. Expressway management system and management regulations should be uniform, and management force should be enhanced. By combining basic features and current situation of expressway operation management, it is required to establish a management system which integrates tool management, road administration management, efficiency management and maintenance management so as to improve expressway management level.

Secondly, make expressway traffic safety monitoring countermeasure sound. With the development and progress of science and technology, relatively advanced and sound traffic facilities are also established for expressway. Traffic surveillance and control system is the most important 
traffic management approach. The symbols displayed in the expressway traffic surveillance and control system may be used to guide driver's safety driving and bring convenience for the traffic sector to direct expressway traffic.

Thirdly, strive to develop intelligent traffic. The modern society is developing to intelligentization which is increasingly favored and welcomed by people and brings great convenience for people's life and work. So, after intellectualized reconstruction of expressway traffic, it can not just greatly improve communication capability and safety, but also break through development bottleneck of science and technology on expressway traffic safety and reduce occurance probability of road accidents dramatically.

\section{Conclusion}

In conclusion, it is very necessary to construct expressway traffic safety evaluation model under current situation. The detailed and specific evaluation system and evaluation problems can vividly reflect current situation and safety level of expressway traffic safety. Besides, the factors influencing expressway traffic safety can be found out rapidly according to the evaluation results so as to enhance expressway management level and ensure travel safety. In one word, the construction of expressway traffic safety evaluation model is of great practical significance.

\section{References}

[1] Chen Jie, Hu Qizhou, Zhu Yun, Comprehensive evaluation model of freeway traffic safety considering subjective and objective factors, China Safety Science Journal, 2017-05-15.

[2] Zhu Mingming, Study on Comprehensive Assessment and Measurements of Expressway Traffic Safety, Chongqing Jiaotong University, 2015-04-25.

[3] Wu Xinru, Chen Heshui, Yang Qianlong, Highway traffic safety properties comprehensive evaluation model, Shanxi Architecture, 2013-04-20.

[4] Zhao Xinyong, Freeway Traffic Safety Evaluation Method Based on Multi-source Heterogeneous Data, Harbin Institute of Technology, 2013-09-01. 